\title{
Refractory Diffuse Large B-Cell Lymphoma
}

National Cancer Institute

\section{Source}

National Cancer Institute. Refractory Diffuse Large B-Cell Lymphoma. NCI Thesaurus.

Code C8853.

Diffuse large B-cell lymphoma that is resistant to treatment. 